\title{
BARAJAS-PARK. EDIFICIOS PARA INDUSTRIA LIMPIA
}

\author{
(BARAJAS PARK - BUILDINGS FOR CLEAN INDUSTRY. SPAIN)
}

J. Ramón Argelés, Dr. Arquitecto

Dragados y Construcciones, S. A.

MADRID/ESPAÑA

Fecha de recepción: 9.IV.90

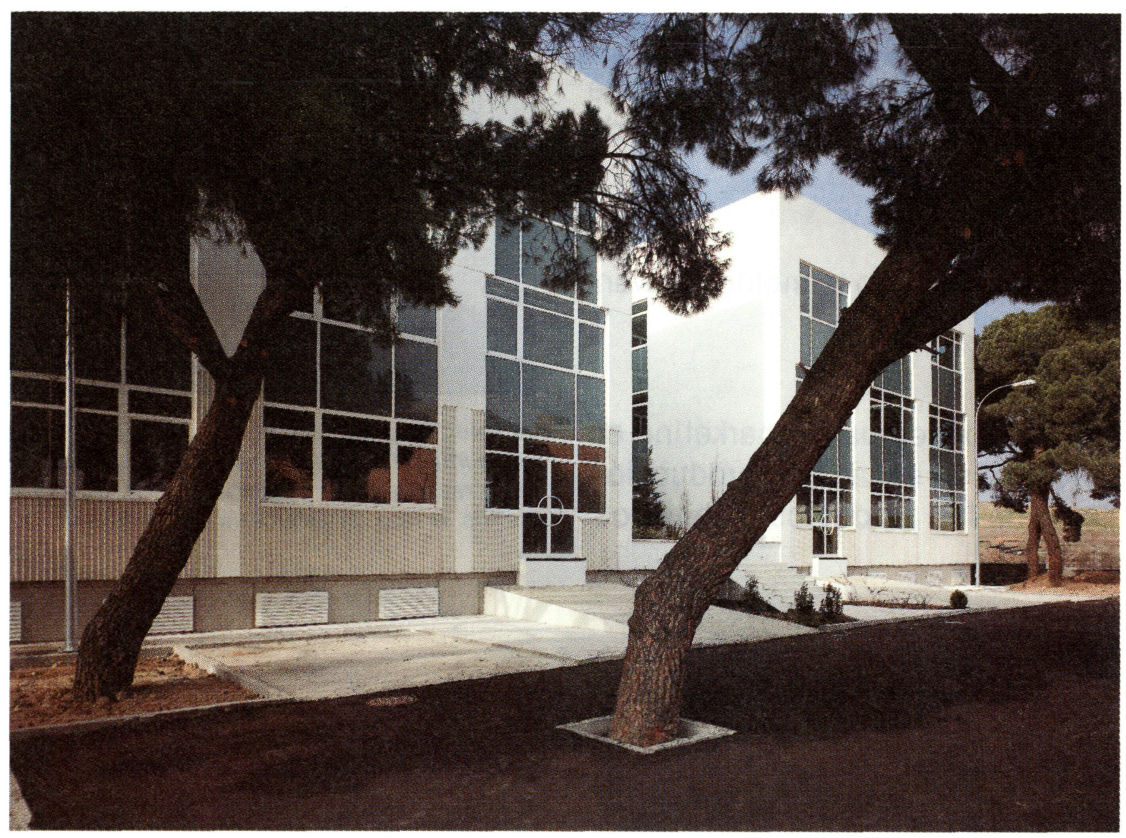

\section{RESUMEN}

En los últimos 20 años ha evolucionado el concepto de Edificios de uso industrial produciendo un importante cambio en los productos inmobiliarios.

En tecnologias avanzadas las diferencias ergonómicas entre industria y servicios tienden a reducirse.

Nuevas Técnicas de Gerencia Empresarial favorecen que alrededor de proyecto o productos se creen equipos compuestos por técnicos y comerciales; para este personal, altamente cualificado, se necesita crear ambientes y entornos agradables.

La función de almacenamiento también ha evolucionado, el volumen de stock se reduce, y a favor de rotaciones rápidas.

El edificio empresarial actualmente se diseña pensando en las interacciones de funciones. La flexibilidad de los espacios traduce un proceso de diversificación económica.

Se trata de proyectar edificios que acojan empresas muy diversas con idéntica eficacia.

Facilidad de evolución y desarrollo de las empresas, con fáciles modificaciones, optimización de comunicaciones, externas e internas con previsión de aparcamientos.

Fácil incorporación de instalaciones, diversas y sofisticadas. Un edificio de estas caracteristicas ofrece: Buena ubicación. Buena comunicación: Mucha luz natural. Fachadas de calidad arquitectónica. Buen aislamiento. Superficies diáfanas interiores. Capacidad de incorporar instalaciones y servicios sofisticados. Paisajismo y vecindad de calidad.

\section{SUMMARY}

During the last 20 years the concept of BUILDINGS FOR INDUSTRIAL USE has envolved and has produced an important change in REAL ESTATE PRODUCTS.

The ergonomic differences between INDUSTRY and SERVICES tend to decrease as far as technological advances are concerned.

New Company Management Techniques faver the creation of technical and commercial teams to study projects and products and this highly-qualified personnel requires a comfortable atmosphere and surroundings.

Warehousing has also progressed. Stock volumes have been reduced in favor of faster rotation.

At present, commercial buildings are designed with interaction functions in mind. The space's sensitivity is translated in a economic diversification process.

The objective is to design buildings that attract different types of companies, offering all of them identical efficiency.

Facility for development and evolution of the companies, with simple modifications, optimizing internal and external communications, with provisions for parking.

Facility for incorporating diverse and sophisticated services. A building with these characteristics offers: A good location. Good communications. Good natural lightinc. High-quality structural desing. Good insulation. Large open interior spaces. Capacity to incorporate sophisticated installations and services. Attractive scgnery/views and a good neighborhood. 
En los últimos años se ha notado en América y luego en Europa una evolución muy rápida de las empresas que se caracteriza por:

- El abandono progresivo de muchas funciones de producción primaria para actividades limpias de montaje y servicios.

- La prioridad a la búsqueda y uso tecnológico avanzadas.

- La integración de los conceptos de marketing en cada etapa del proceso de desarrollo, producción y servicio al cliente de los productos y servicios.

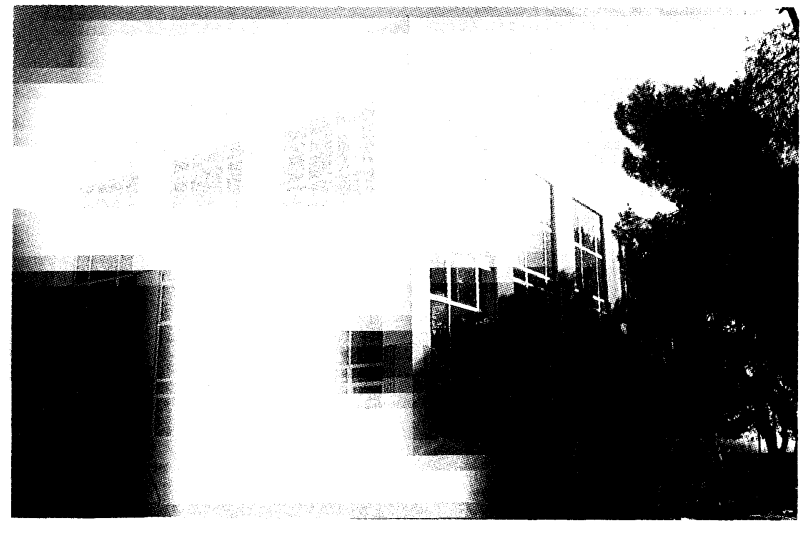

La solución urbanistica y paisajismo, cara a la creación de un medio y una imagen caracteristica, exigió un estudio de la vegetación exis. tente respetando y potenciando los árboles existentes y adecuando a ellos el tipo de jardinería complementaria. a b

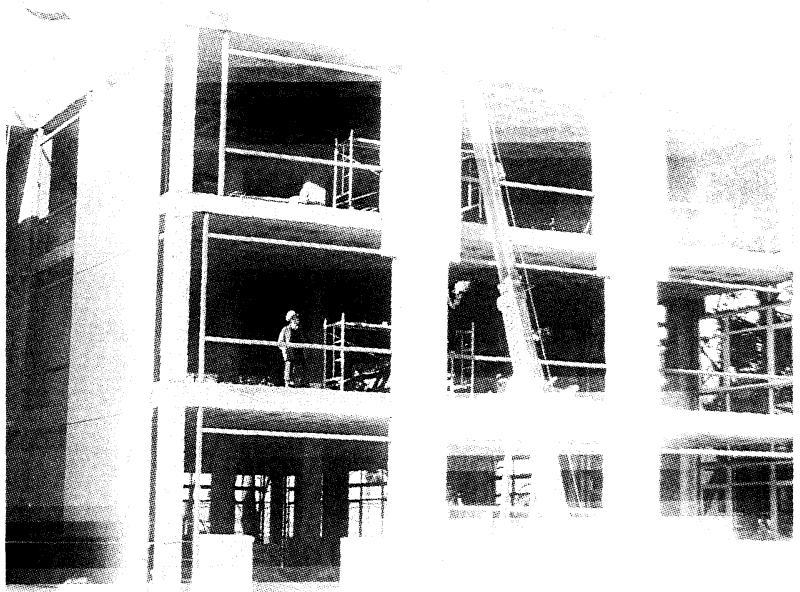



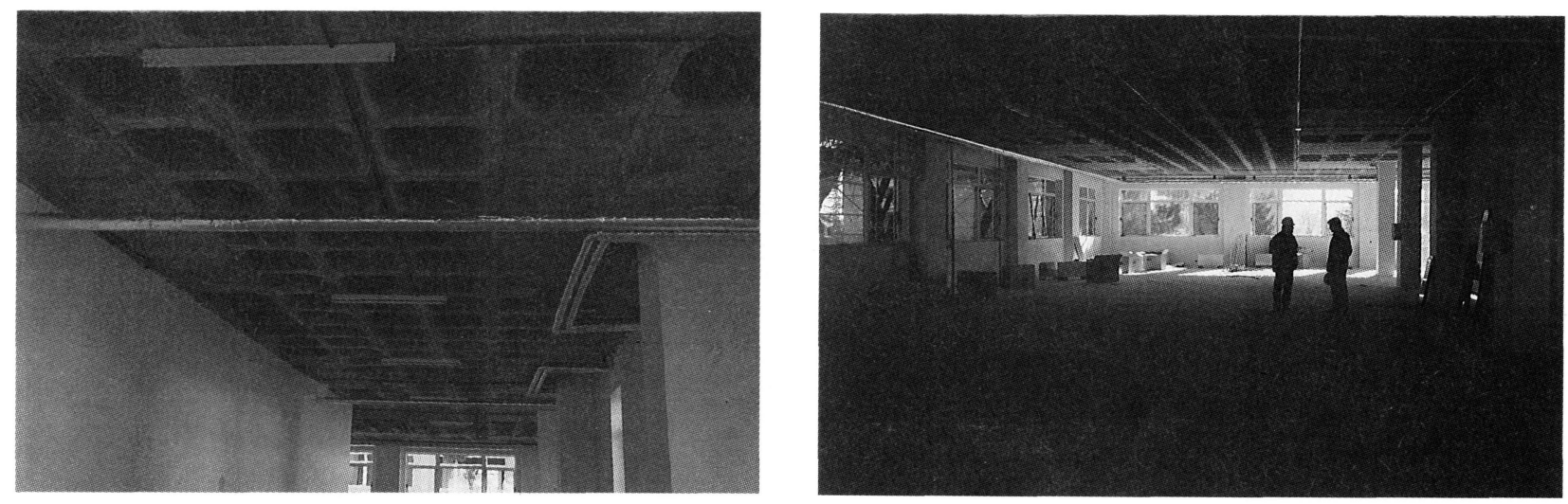

La planta libre de 10,80 m de luz se soluciona estructuralmente con un forjado reticular estándar de $35.45 \mathrm{~cm}$ de canto, según cargas, apoyado sobre pilares de hormigón armado con una distancia entre ejes de 5,40 m y 10,80 m.

C

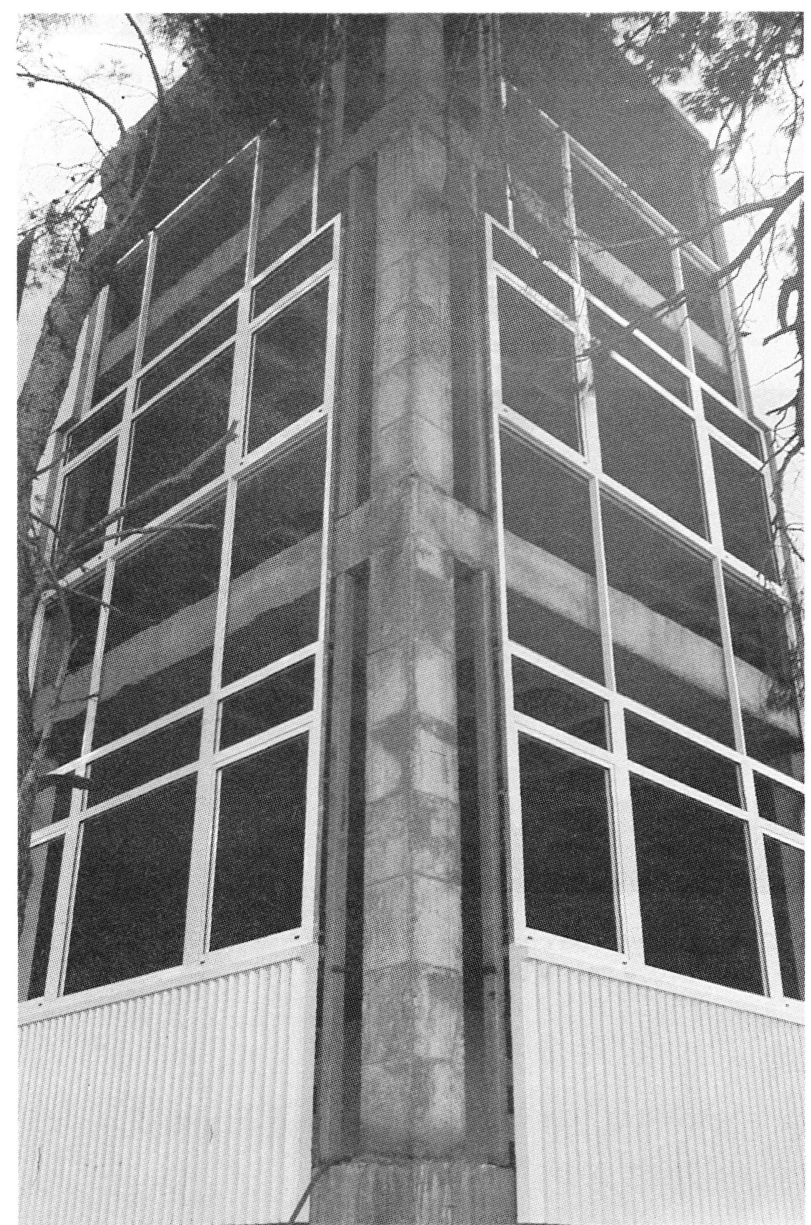

(C) Consejo Superior de Investigaciones Científicas Licencia Creative Commons 3.0 España (by-nc)
Estas evoluciones, muy rápidas, han significado un cambio profundo de los productos inmobiliarios para uso empresarial.

En materia de tecnologías avanzadas, las diferenciás ergonómicas entre el terciario y el secundario, tienden a reducirse. La proporción de estudios, investigación e ingeniería en cada fase de un proceso de fabricación aumenta. Nueva técnicas de gerencia empresarial favorecen la creación, alrededor de un proyecto o un producto, de equipos autónomos compuestos tanto por especialistas comerciales como por técnicos e ingenieros.

Asimismo se nota, para este personal altamente cualificado, la necesidad de un ambiente y un entorno agradable, tanto dentro como fuera del edificio.

\section{a, b y c}

La geometria de los paneles de GRC se proyectó de forma sencilla, compatibilizando grandes volúmenes y composición de huecos, fa. cilitando la percepción de las grandes masas bases del diseño, con la observación del detalle de carpinteria y acabado terso con el ranu. rado, que refuerza y estabiliza conceptualmente la base de los edificios. 

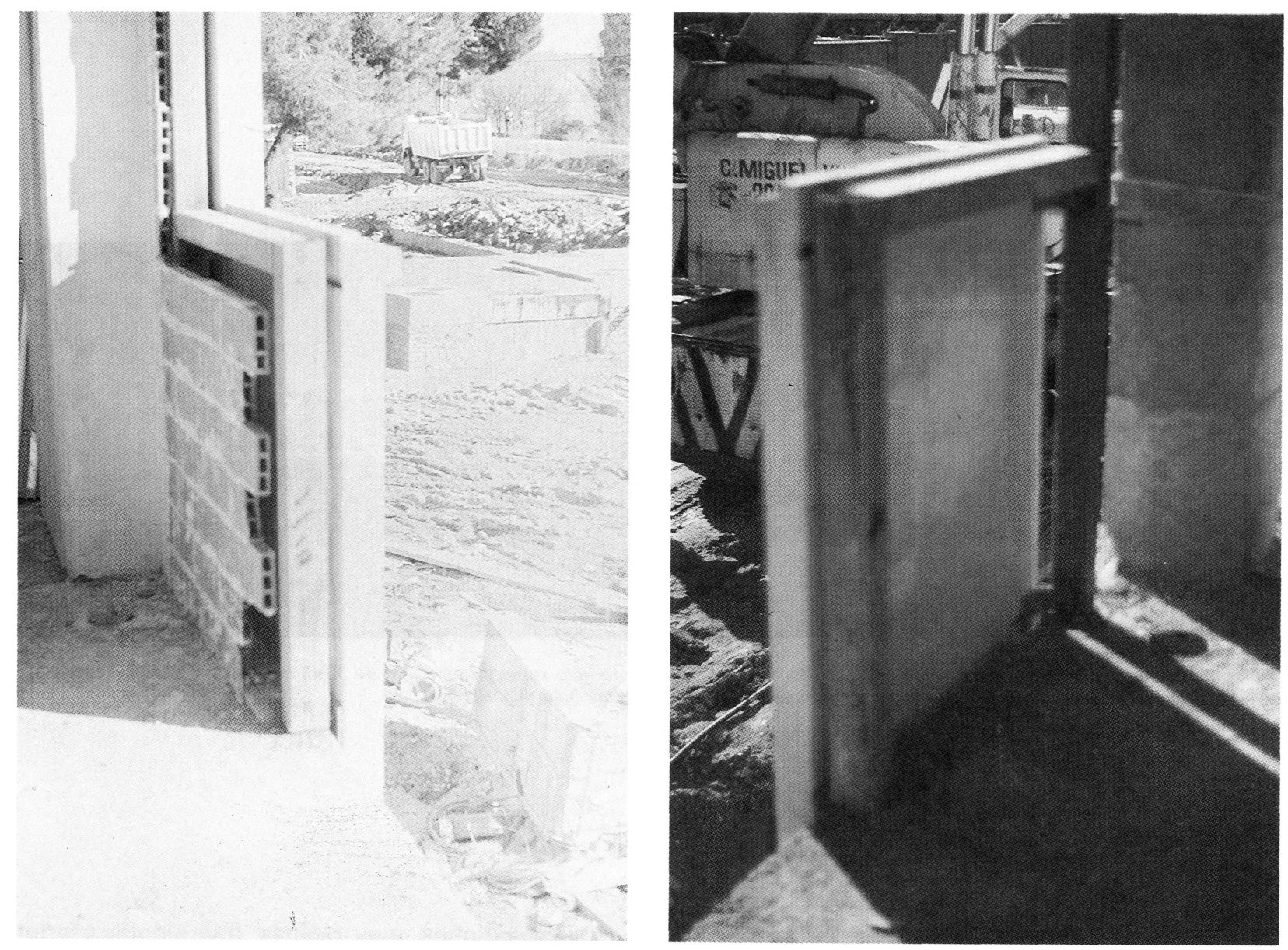

El cerramiento, tratado como cáscara, se compone de un prefabricado de GRC de una sola hoja con aislamiento incorporado. cámara de aire y trasdosado interior opcional de placas de yeso-cartón, o tabiqueria cerámica.

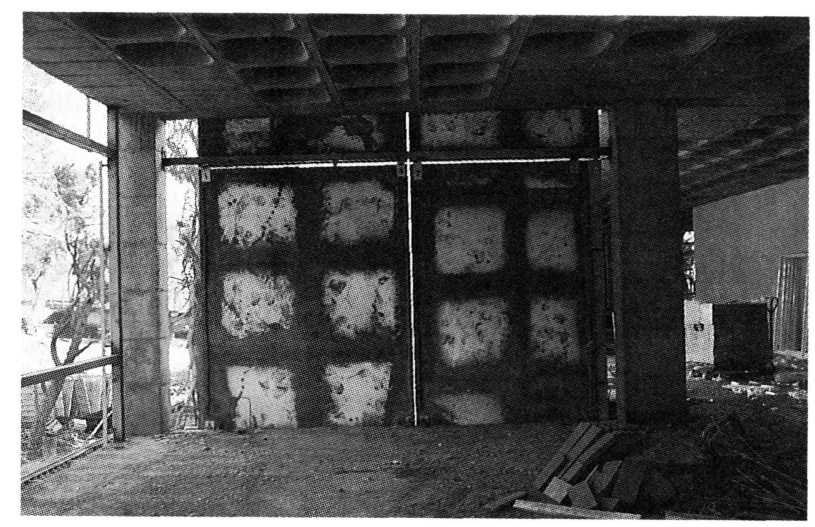

La junta entre paneles, de espesor 10-12 $\mathrm{mm}$, se sella con cordón de fondo de célula cerrada y enmasillado con silicona neutra de color blanco. 


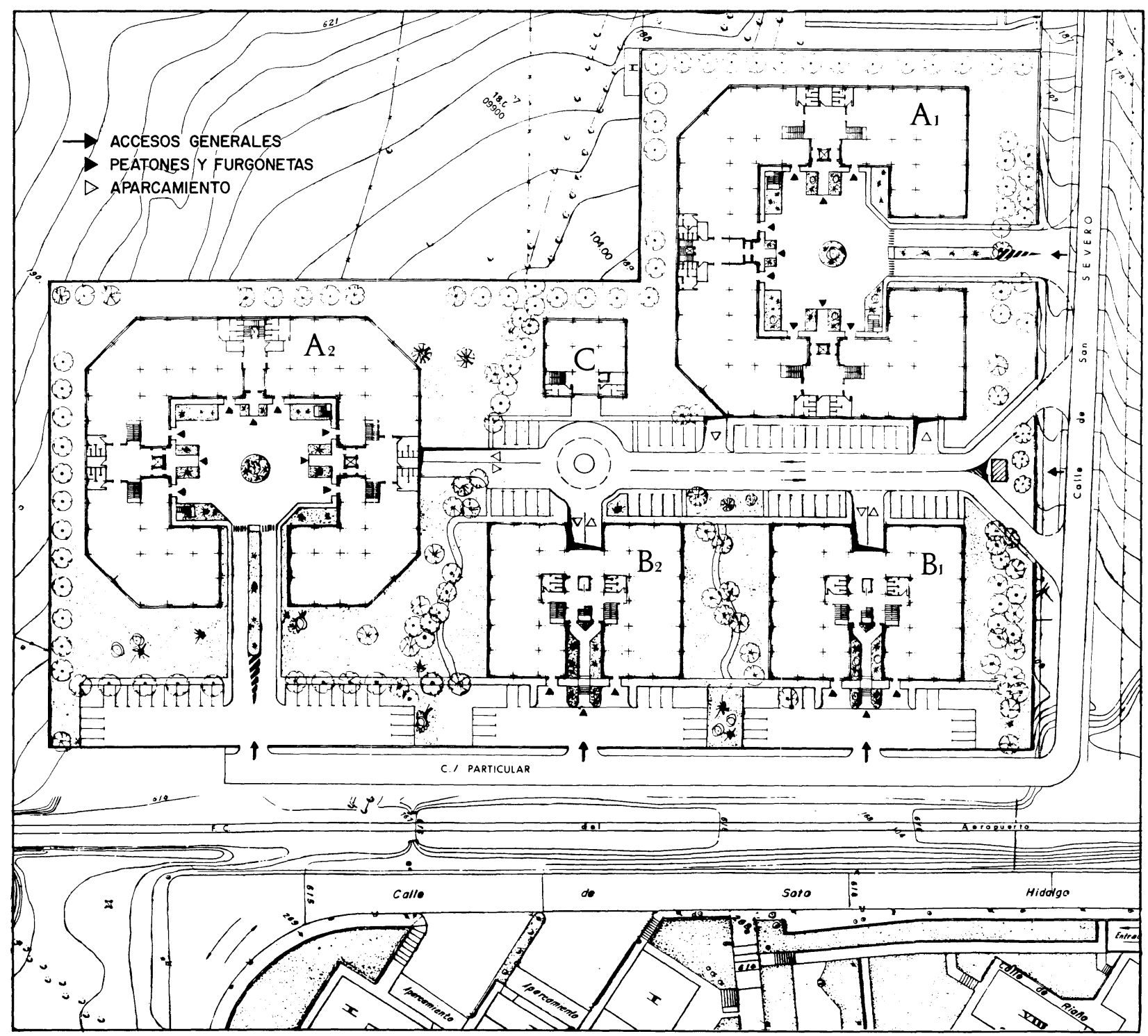

El conjunto de 5 edificios define un recinto central rodeado por 2 edificios de grandes dimensiones (A1 y A2), 2 edificios de tamaño medio (B1 y B2) y un edificio pequeño (C) de servicios comunes.

La composición está generosamente ajardinada y al proyectar la situación de los edificios se tuvo muy presente la situación de los árboles existentes. 


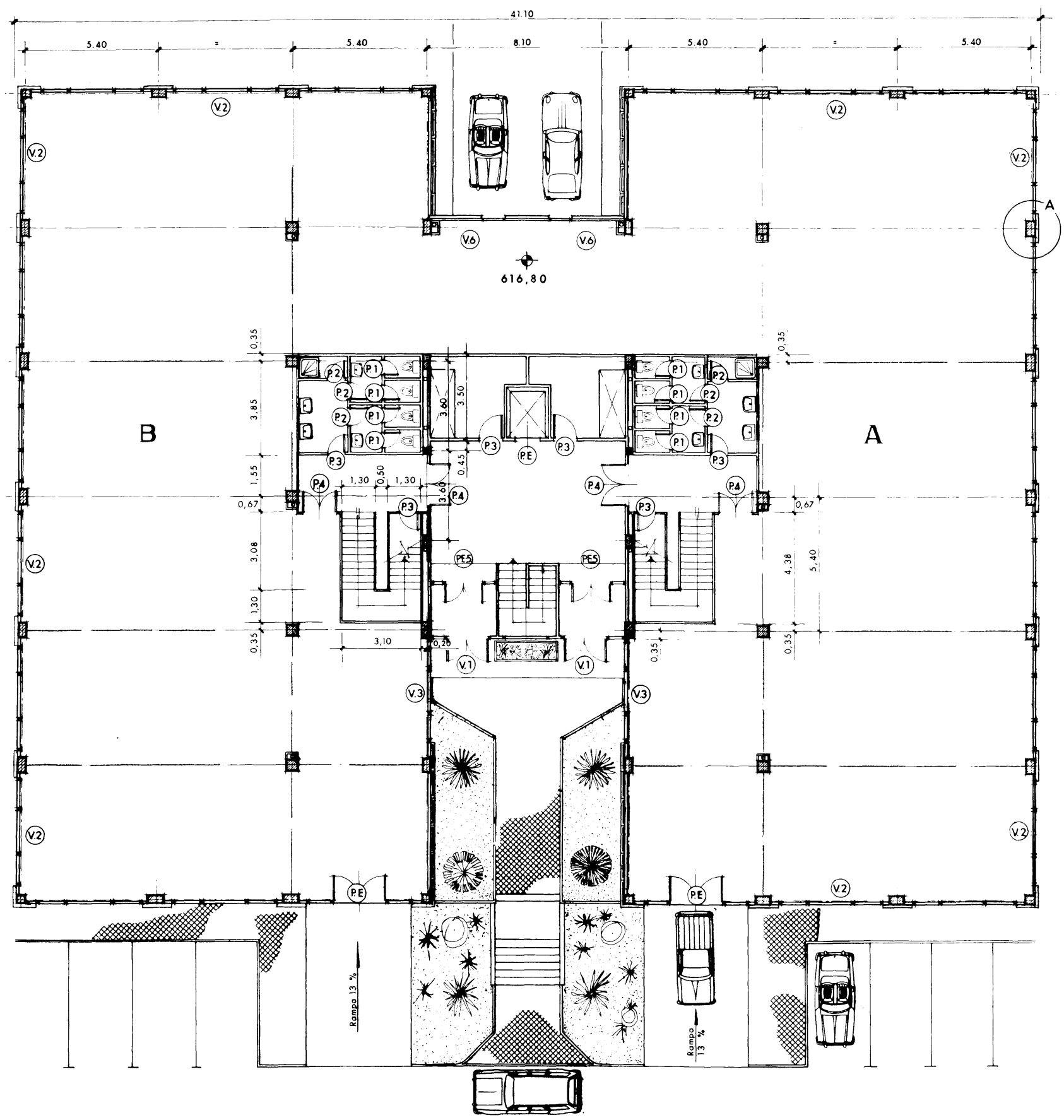

Edificio B1. Planta baja

La trama de 5,40 y 10,80 en la planta libre permite la distribución modular de usos. en zonas de trabajo y pasillos, facilitando la compartimen. tación que se desea, 0,30/0,90/1,20. 


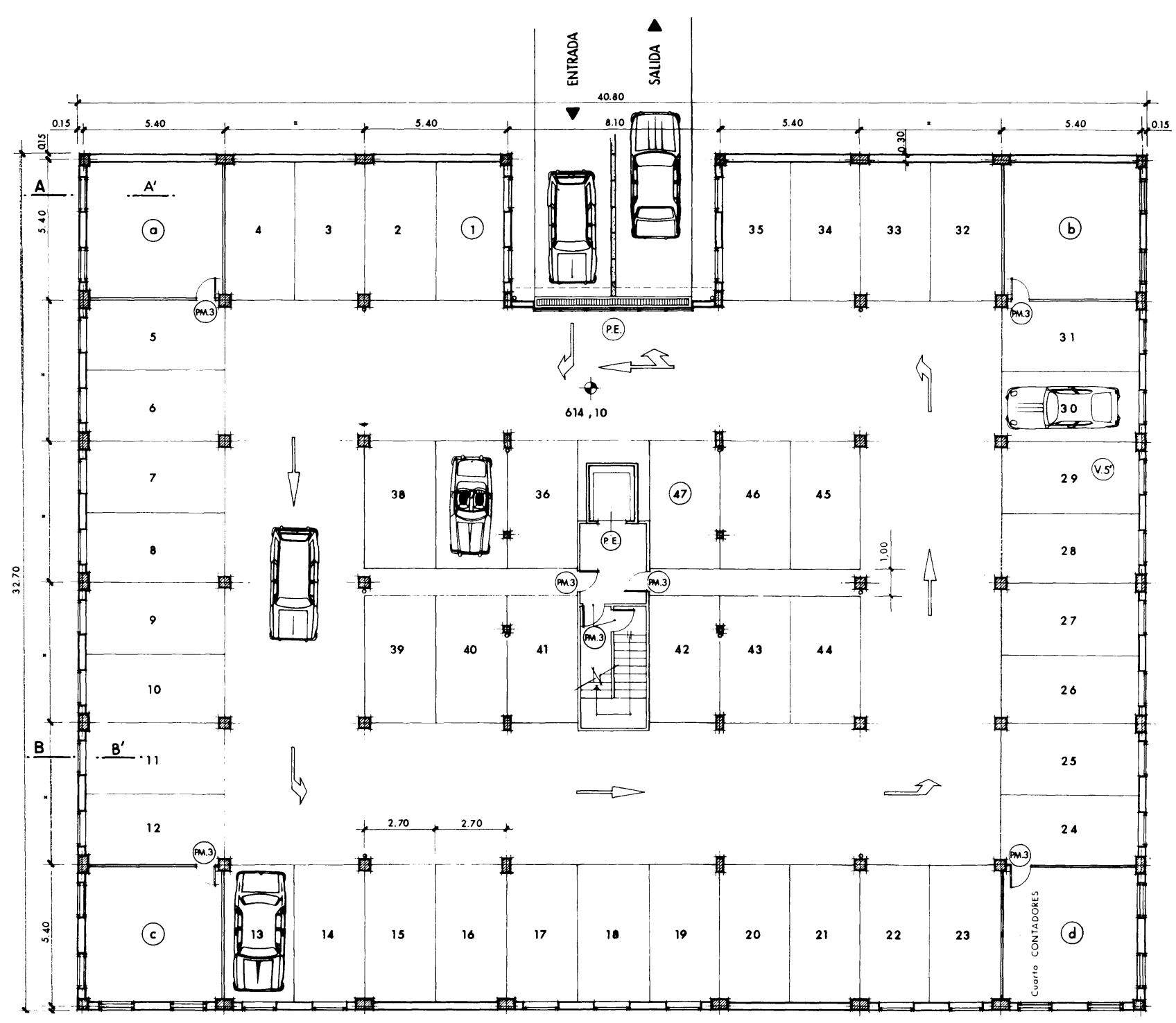

Edificio B1. Semisótano-garaje 


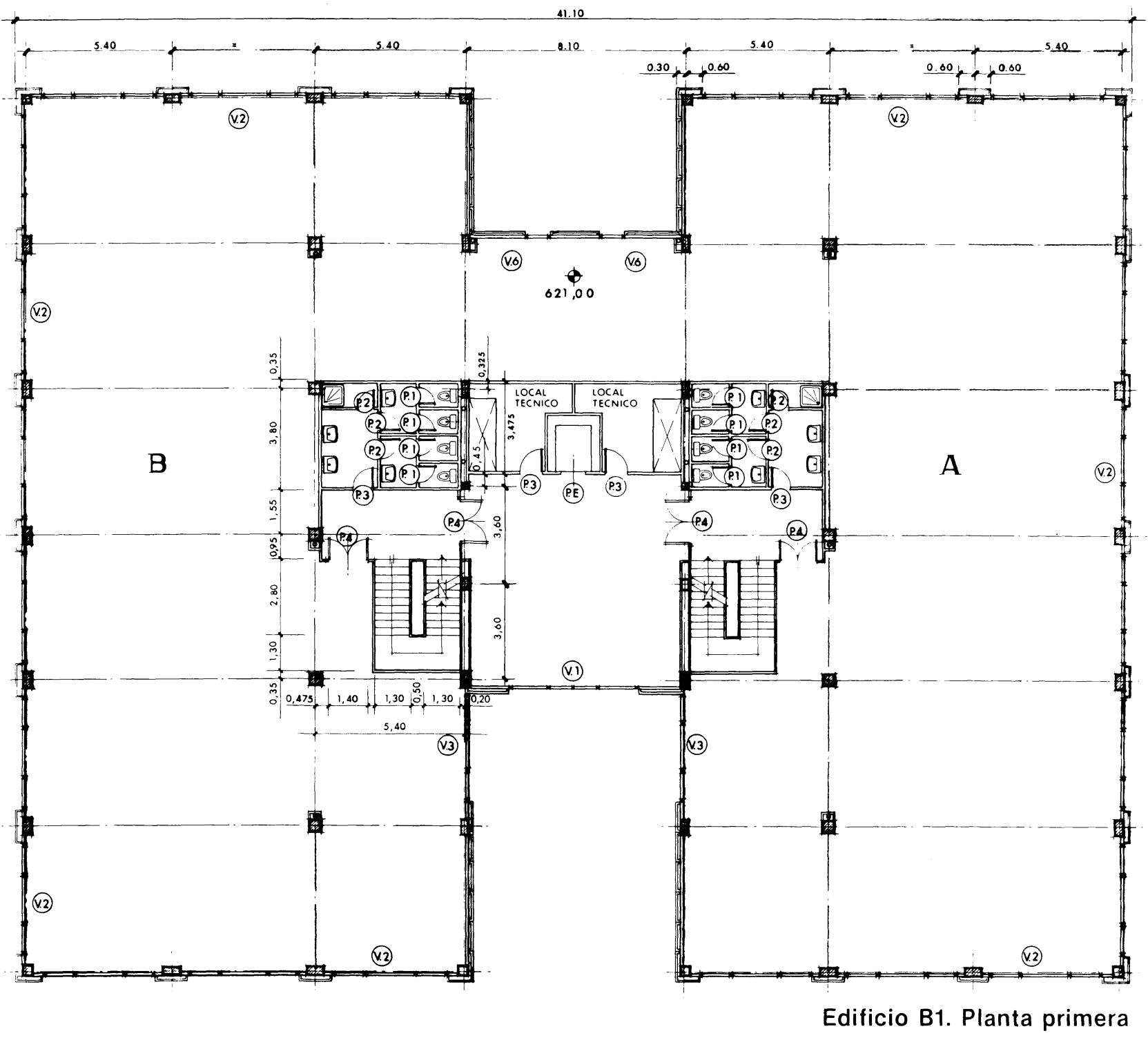




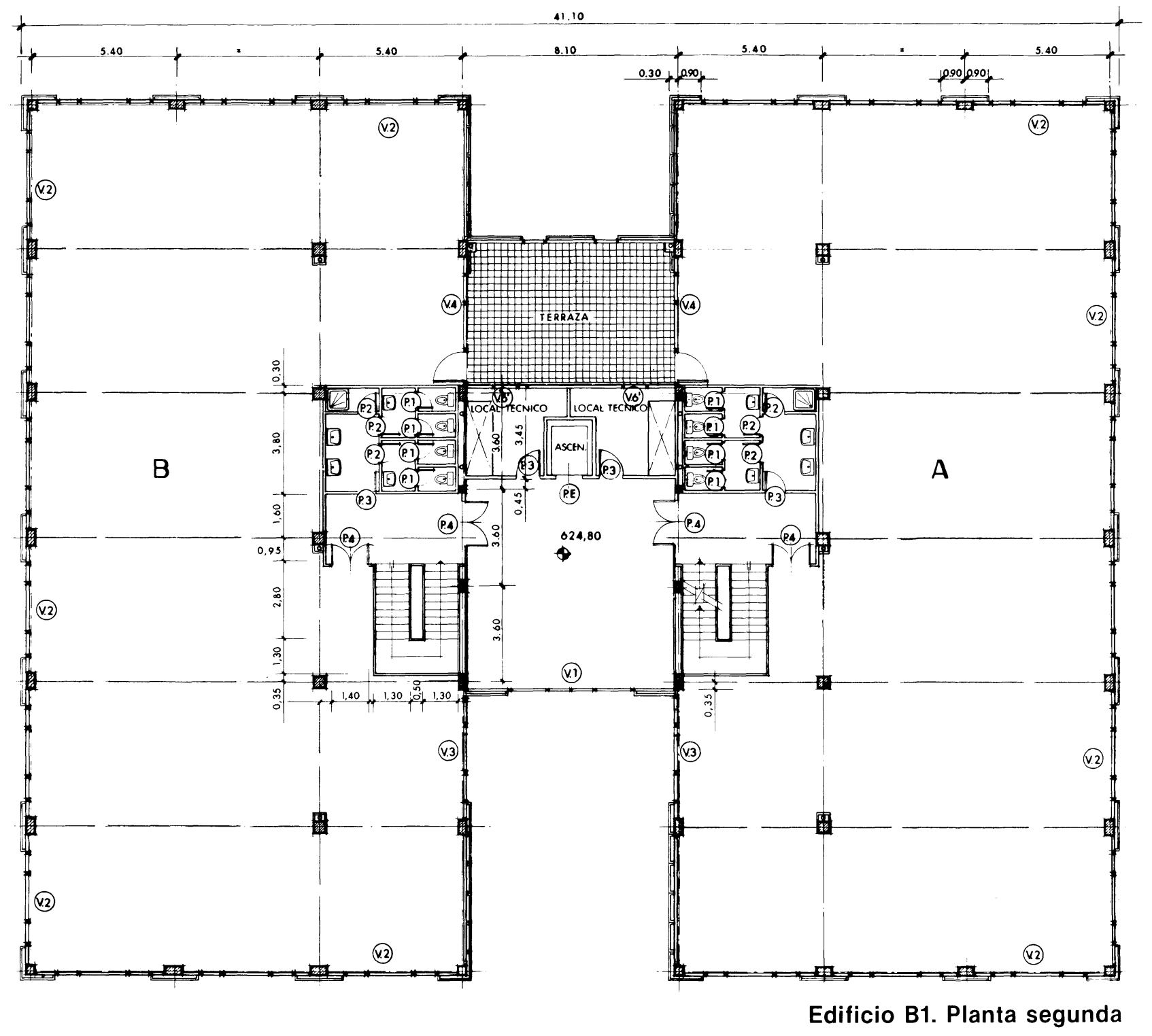




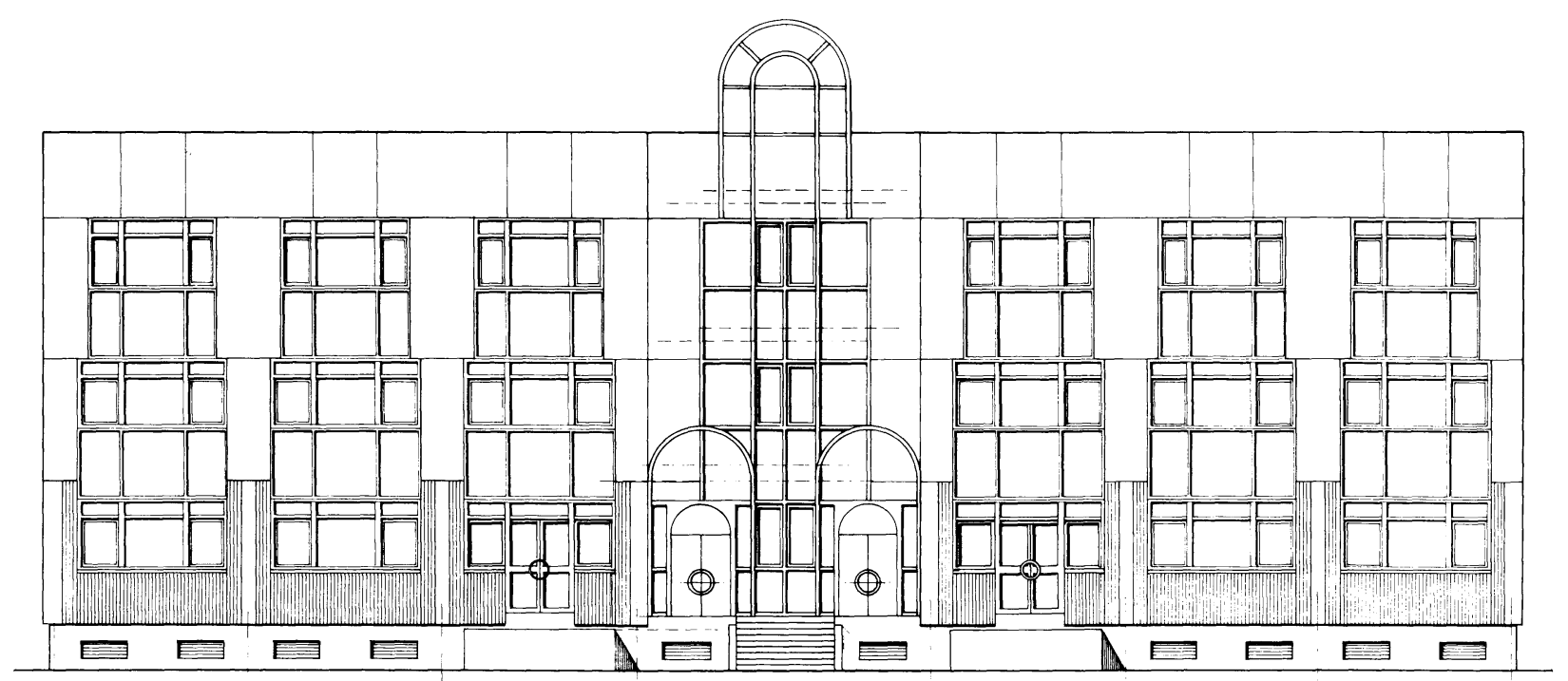

Alzado S.E.

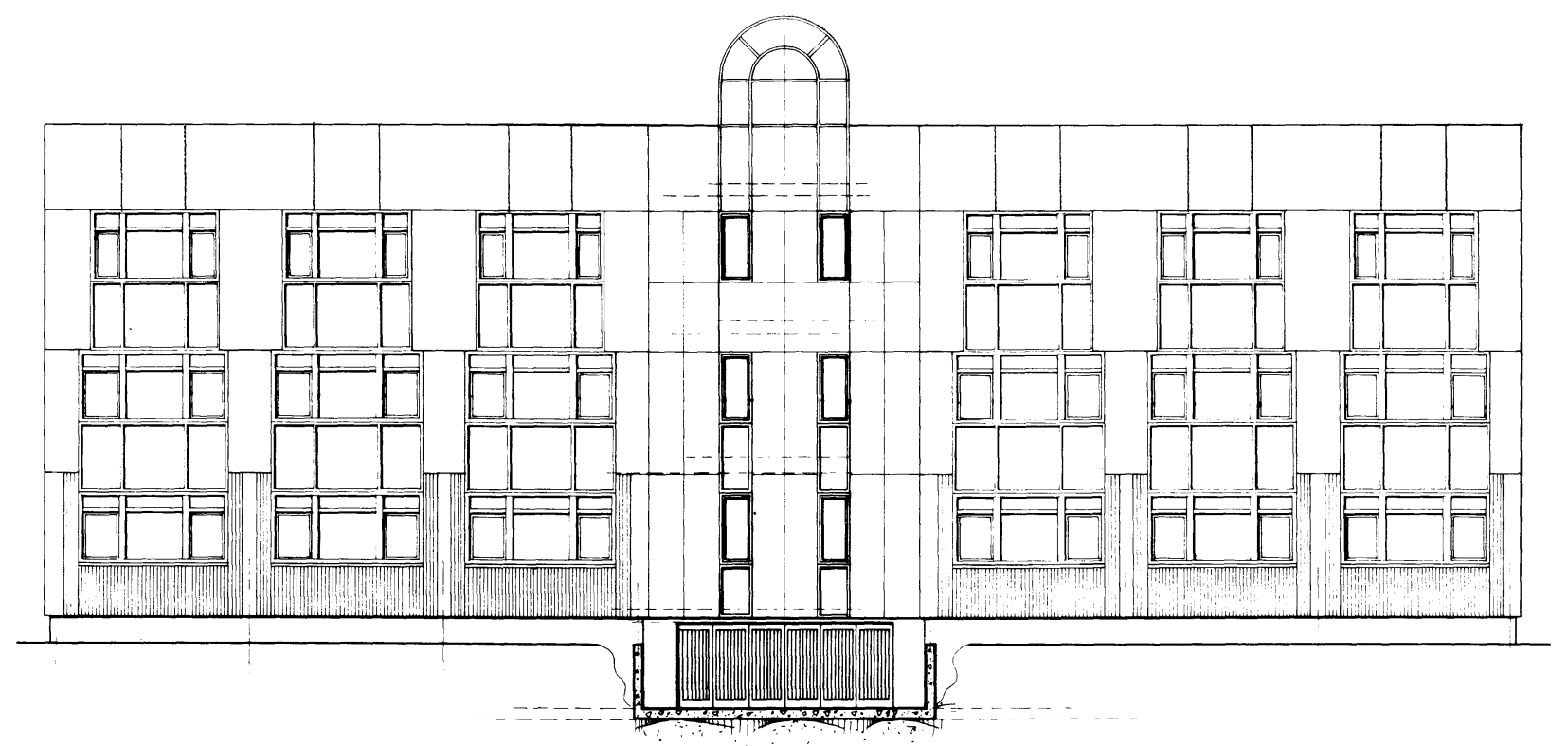

Alzado N.O. 

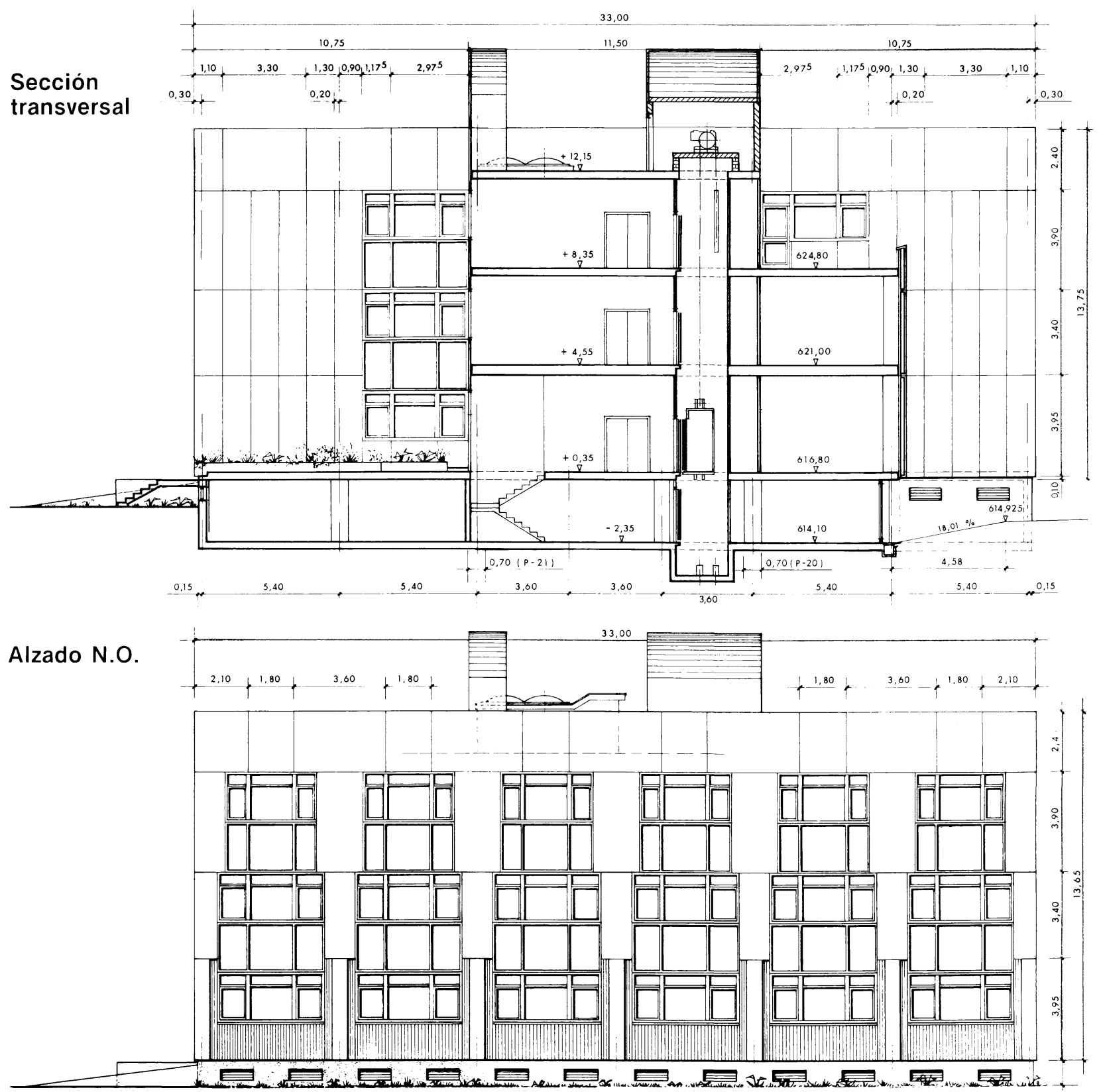
Informes de la Construcción, Vol. 42 n. ${ }^{\circ} 410$, noviembre/diciembre, 1990

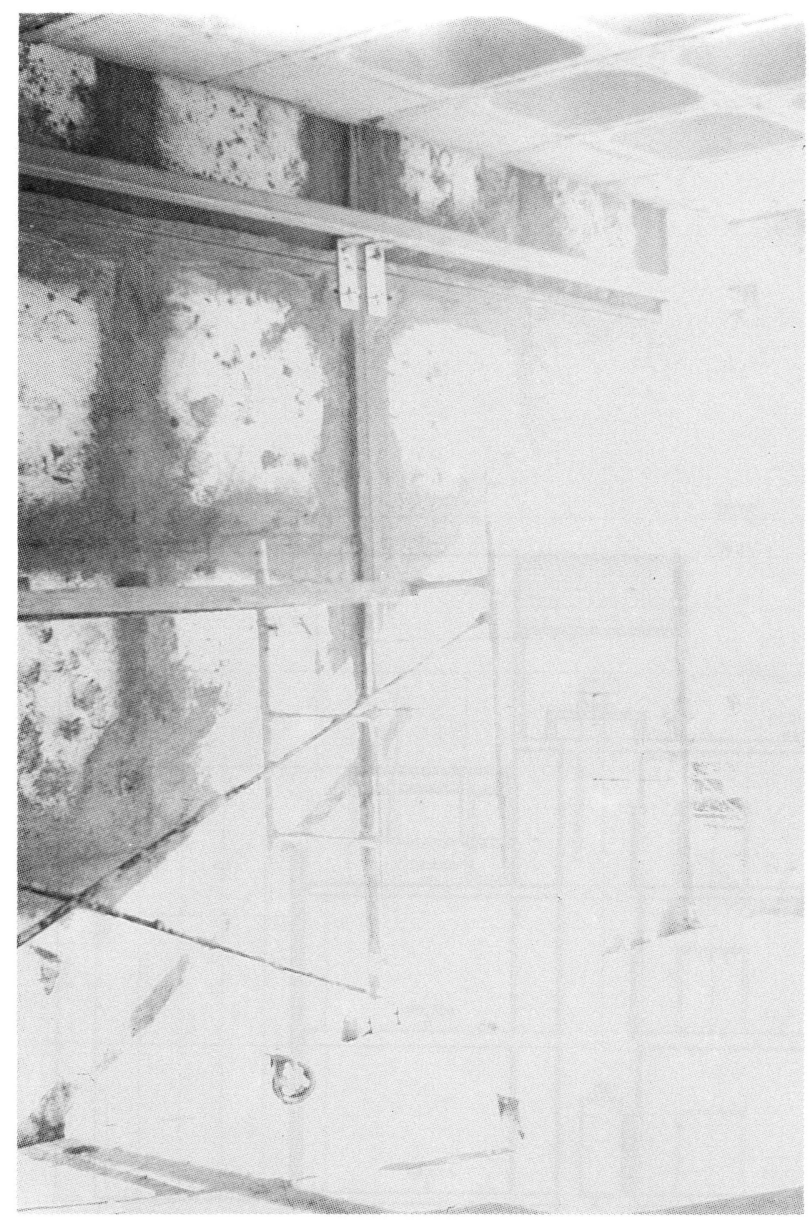

A

C
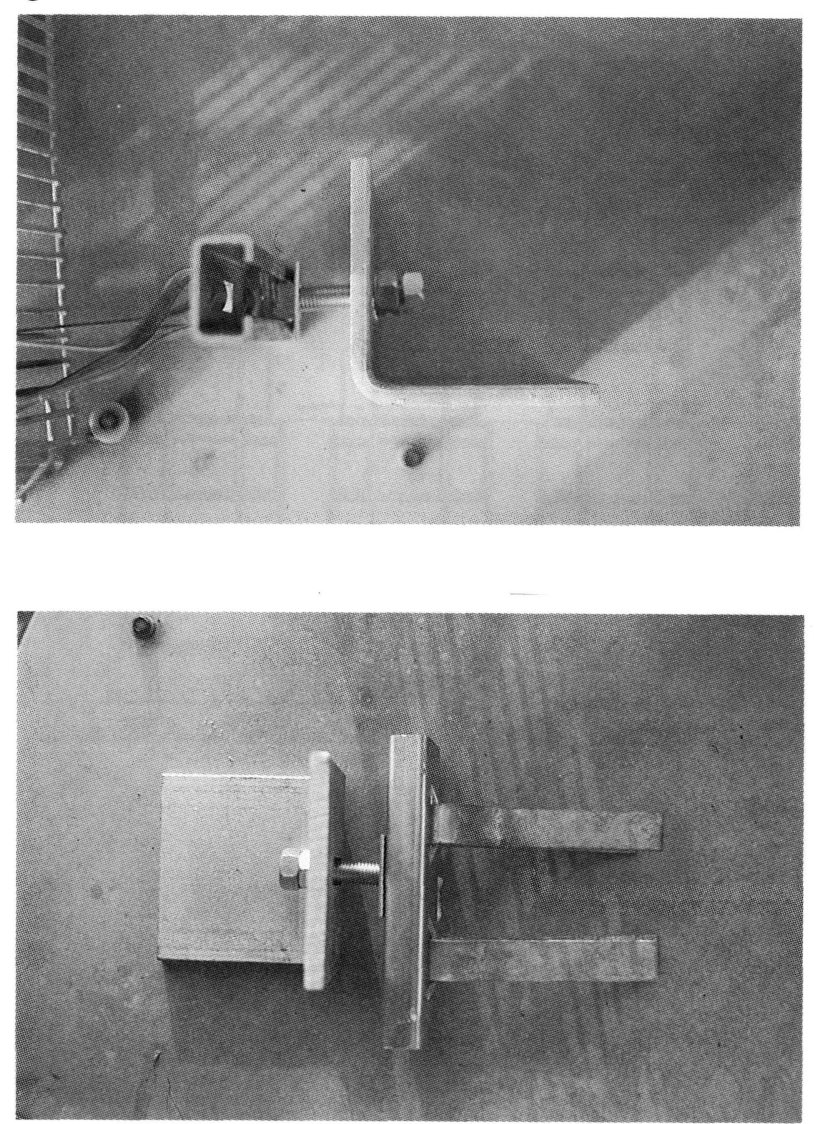

(C) Consejo Superior de Investigaciones Científicas Licencia Creative Commons 3.0 España (by-nc)

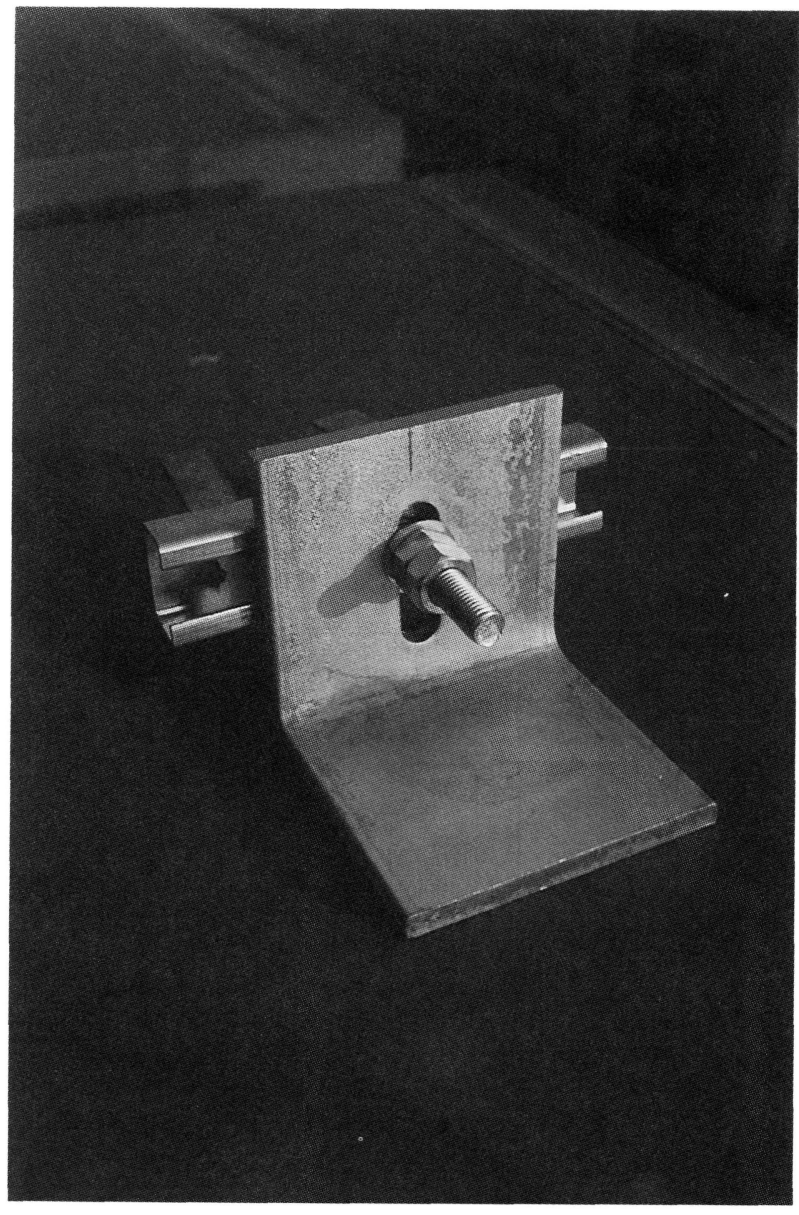

B

Sistema de anclaje empleado en los paneles de GRC:

A. Guia empotrada en el panel que permite el ajuste horizontal paralelo a plano de fachada.

B. Angular de anclaje y regulación horizontal perpendicular al plano de fachada.

C. Espárrago roscado y cabeza giratoria que se adapta en la guía.

D. Arandelas y tuercas de fijación, estabilización y reparto de esfuer. zos.

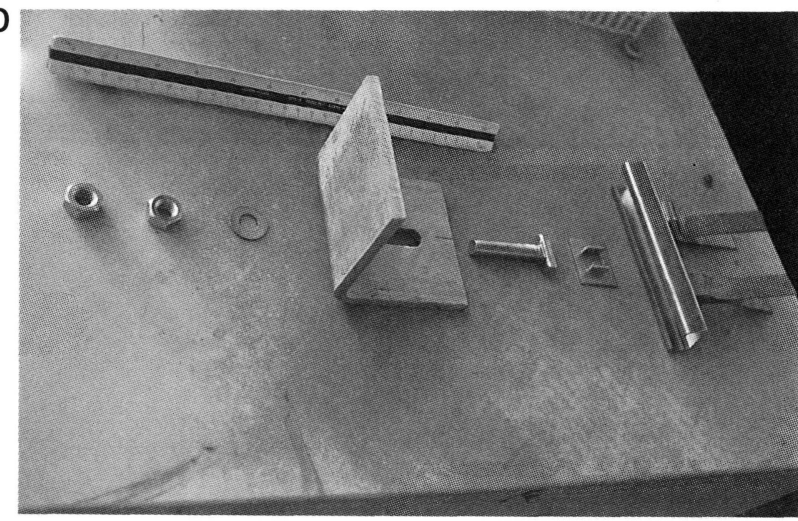

http://informesdelaconstruccion.revistas.csic.es 

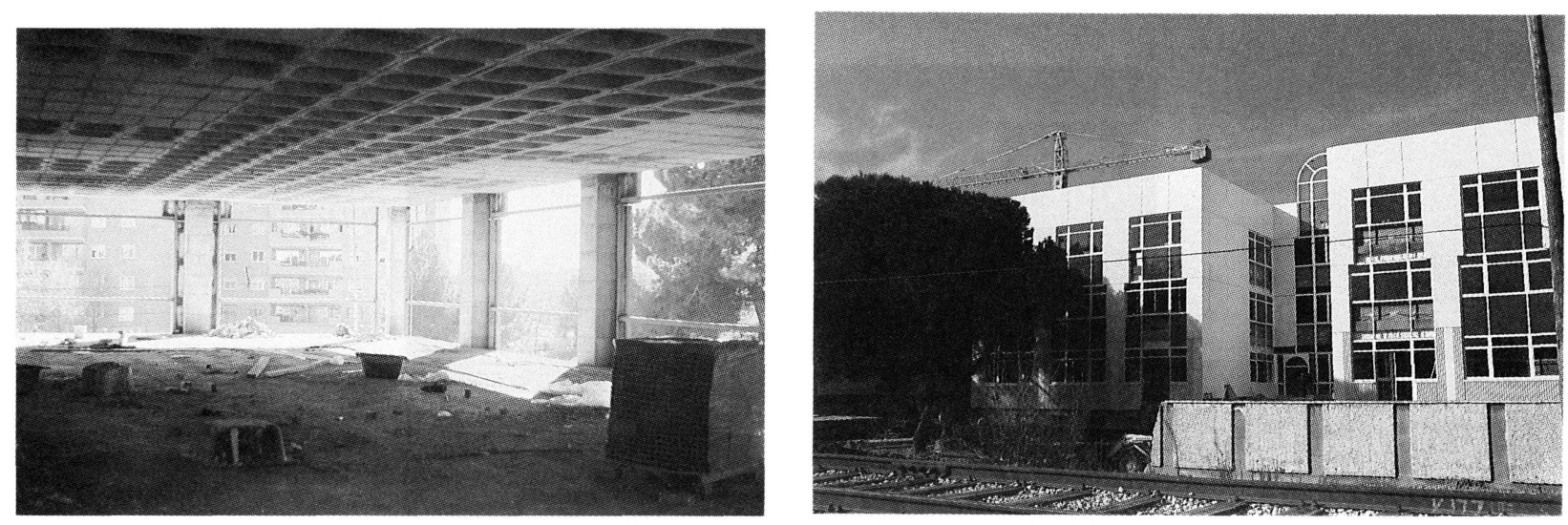

La carpinteria de los huecos es de aluminio lacado con rotura de puente térmico, y se ancla directamente a una estructura portante perime. tral de tubo de acero que absorbe los esfuerzos estáticos y dinámicos y que define los antepechos previamente a la colocación de éstos.

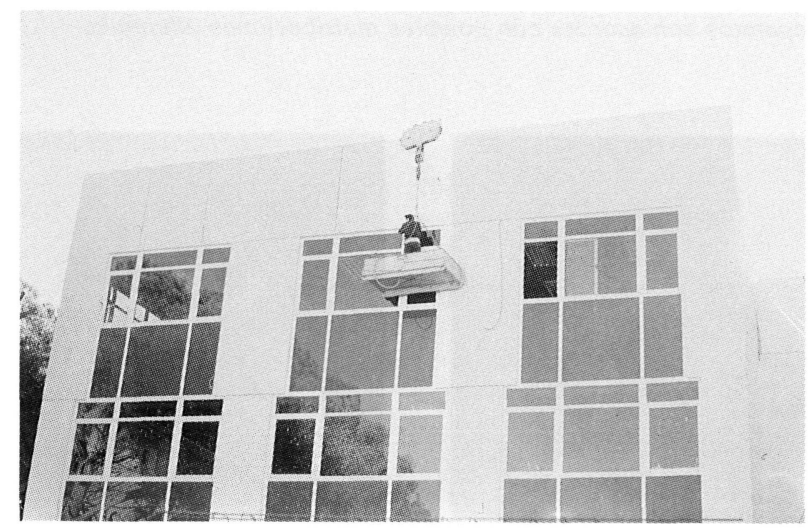

El acristalamiento es en todas las plantas tipo Climalit con luna Parsol verde en su cara exterior y en planta baja sustituido por cristal Stadip en color verde; los antepechos del acristalamiento se resuelven con cristal opaco color verde con placas aislantes en su trasdós.

Ha evolucionado también muy rápidamente la función de almacenamiento, ya que los productos se hacen más pequeños, y el volumen del stock se reduce a favor de rotaciones más rápidas. La tipología de estos locales ha tenido que adaptarse de manera general. El edificio empresarial ya no se diseña en torno a un concepto único (torre de oficinas, taller diseñado alrededor de las máquinas, almacén), sino tomando en cuenta la interacción de varias funciones. La flexibilidad del edificio traduce un proceso muy rápido de diversificación económica.

(C) Consejo Superior de Investigaciones Científicas Licencia Creative Commons 3.0 España (by-nc)
Las palabras clave para esto son: flexibilidad, usos múltiples, adaptabilidad, módulos; pero también comunicación, equipo, calidad de vida, tecnología.

Dentro de este marco los edificios llamados "high tech" van al extremo de esta evolución: no solamerite relacionan entre ellas sus superficies de usos diferentes, sino que pretenden ofrecer un espacio en el cual podrán desarrollarse actividades en plena evolución con gran flexibilidad para variar los usos y funciones de las varias superficies que lo componen.

http://informesdelaconstruccion.revistas.csic.es 


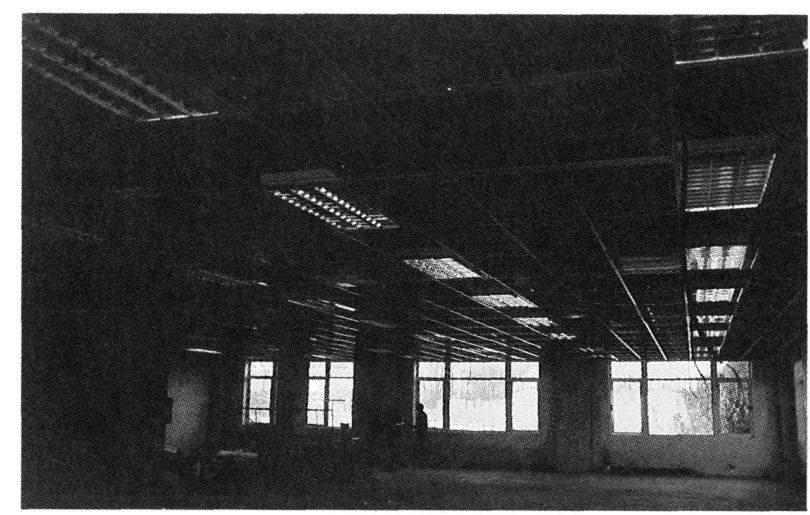

El techo va equipado con instalaciones base vistas, con posibilidades de ampliación y de ocultación.

En planta superior de oficina se dota de falso techo acústico con pla fones empotrados de alumbrado fluorescente, con nivel medio de 500 lux, al que se incorporan rendijas de impulsión del aire acondicionado también se empotran en falso techo las rejillas de extracción de aire y se sitúan los detectores de incendios.

La disposición de todo el conjunto es modular: redes. circuitos y aparatos son acordes con posibies distribuciones diferentes

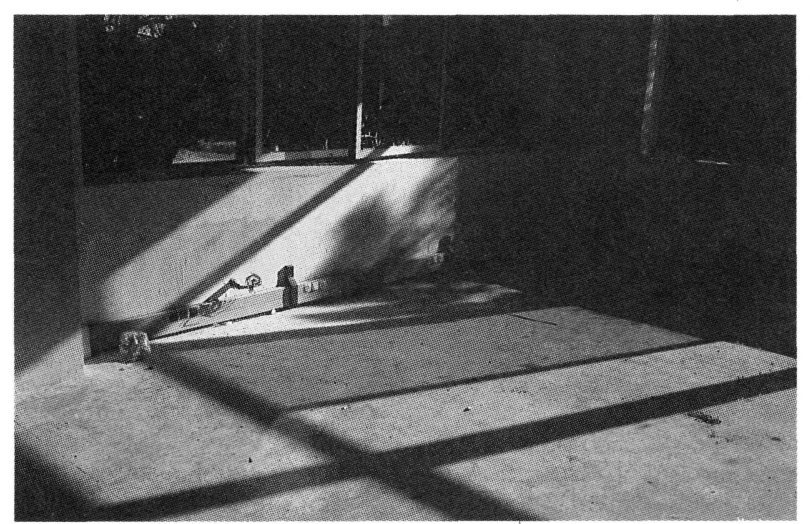

En el rodapié perimetral técnico se alojarán las posibles distribucio nes de energía eléctrica de luz y fuerza, asi como el cableado telefónico e informático.

Se trata entonces de locales de usos múltiples que tienen la facultad de:

- Acoger empresas muy diversas.

- Acoger usos y funciones muy diversas con la misma eficacia.

A la fecha, las empresas que usan este tipo de edificio, caen en las siguientes categorias:

\section{Informática y peri-informática}

- Centro de cálculo y de servicios informatizados.

- Ordenadores centrales.

- Distribución en time-sharing de informática.

(c) Consejo Superior de Investigaciones Científicas Licencia Creative Commons 3.0 España (by-nc)

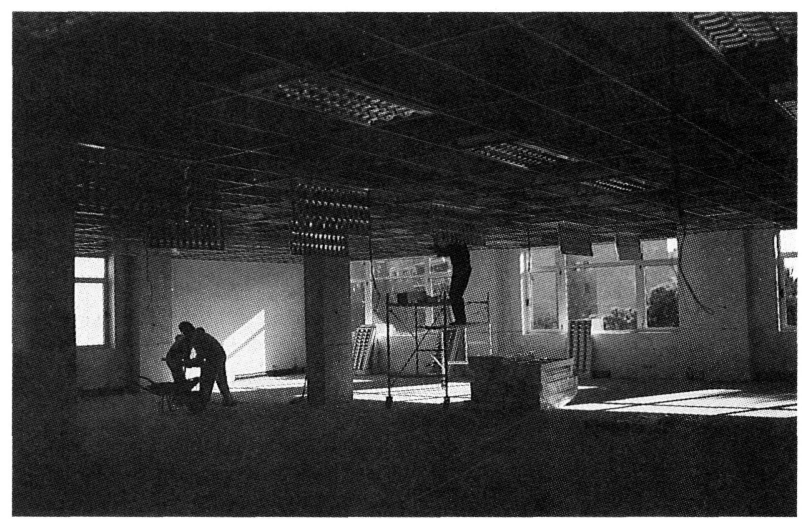

El acabado del suelo de planta baja y $1 .^{a}$ es tipo industrial a base de acabado de hormigón con tratamiento antidesgaste y antipolvo, dándose con acabado de moqueta la planta $2 .^{a}$ de oficinas, pero estudiado para posible cambio de otros acabados, incluidos suelos flotantes para informática.

- Talleres de realización y producción de software.

- Telecomunicación y teléfono.

\section{Farmacia/Biologia/Quimica}

- Laboratorios de investigación y producción ligera.

- Ingeniería genética.

- Producción de aparatos médicos altamente sofisticados.

\section{Tecnología avanzada, industrial y electrónica}

- Robótica.

- Automatismos.

- Instrumentos de medición y control. http://informesdelaconstruccion.revistas.csic.es 


\section{Actividades comerciales ligadas a productos avanzados}

- Centro de servicios post-venta y mantenimiento.

- Salas de exposición y demostración profesional.

- Bancos de pruebas y ensayos.

- Formación de los clientes al uso de los productos o sistemas.

\section{Almacenamiento-Repuestos}

Es interesante destacar que las empresas de estas categorías buscan una flexibilidad máxima del edificio en una etapa muy específica de su desarrollo, es decir, cuando alcanzan un tamaño medio de 30 a $50 \mathrm{em}$ pleados. Al mismo tiempo otorgan mucha importancia a la promoción de su imagen profesional, lo que les induce a buscar locales de calidad en su arquitectura y entorno.

Además la necesidad de optimizar las comunicaciones internas y externas, hacen que favorezcan edificios de pocas plantas (1 a 3), y que estén bien provistos de aparcamiento, tanto para los clientes como para los empleados.

En base a estos conceptos, el edificio típico ofrece:

- Una fachada exterior de gran calidad arquitectóni$\mathrm{ca}$, con mucha luz natural y para grandes superficies un patio interior.

- Superficies internas entregadas con un mínimo de equipamiento (local social, recepción, circulación vertical) pero capaces de recibir con intervenciones mínimas los sistemas más sofisticados.

- Un paisajismo y una vecindad de calidad con acceso fácil a todo tipo de vehículos.

- Un aislamiento térmico de calidad del edificio (vidrio doble, aislamiento térmico de las fachadas y techos).

\begin{tabular}{l} 
Equipo Técnico \\
Arquitecto Consultor: Bernard Malabre. \\
Arquitecto: José R. Argelés. \\
Aparejadores: José de San Andrés y José R. Gutiérrez. \\
Promotor: Parques Empresariales, S. A. \\
Gestión y Dirección: Tecnopark. \\
Constructor: Dragados y Construcciones, S. A. \\
\hline
\end{tabular}

$\star \star \star *$

\title{
publicación del ICCET/CSIC
}

\author{
ACUEDUCTOS ROMANOS EN ESPAÑA \\ Carlos Fernández Casado \\ Prof. Dr. Ing. de Caminos, Canales y Puertos
}

Esta publicación se compone de una serie de articulos, publicados en la Revista "Informes de la Construcción", en los cuales se hace un análisis de los acueductos romanos que existen en España y el balance de las condiciones de conservación en que se encuentra cada uno de ellos, incluyendo referencias históricas y literarias. Se ha ilustrado con la reproducción de la valiosa documentación gráfica que posee el prestigioso autor.

Un volumen encuadernado en couché, a dos colores, de $21 \times 27$ centimetros, compuesto de 238 páginas, numerosos grabados, dibujos, fotos en blanco y negro y figuras de línea.

Precio: España, 1.500 ptas., 21 \$ USA.

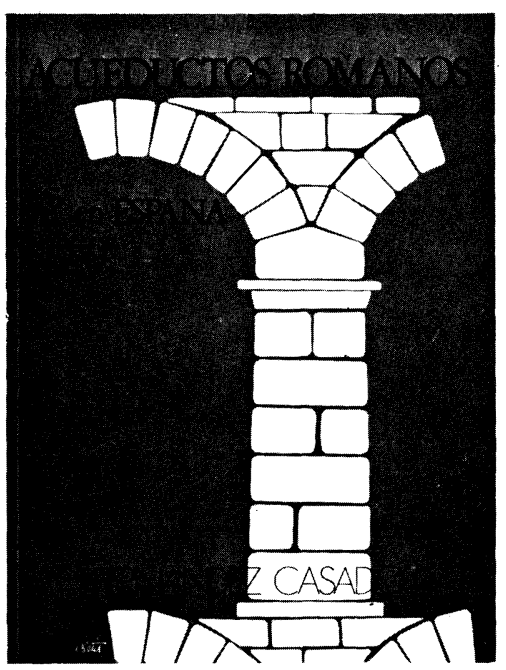

